\title{
Polarized Physics with an Electron-Ion-Collider
}

\author{
Antje Bruell \\ Jefferson Lab \\ 12000 Jefferson Avenue, Newport News, VA 23606 - USA
}

\begin{abstract}
The perspectives of a future Electron-Ion-Collider on spin physics and nucleon structure are presented together with the present design options for such a machine.
\end{abstract}

\section{Introduction}

A great achievement of nuclear and particle physics has been the quantitative verification of the QCD theory in hard scattering processes, at distance scales several times smaller than the size of the proton. At these short distances, the quarks and gluons have a very clear experimental signature, and their dynamics follows the prediction of perturbative QCD calculations. Such experiments have, e.g., established that the quarks carry about $50 \%$ of the proton's momentum (the rest being carried by gluons), and - surprisingly -only $30 \%$ of the proton's spin. However, there are still glaring gaps in our knowledge of quarks and gluons inside the proton. What is the role of gluons and angular momentum in the description of the proton's spin? What is happening at very low momentum fractions where more and more gluons are expected to start overlapping each other? How large are the correlations between quarks and gluons inside the nucleon? And how are they distributed in transverse space?

Recent advances in computational technology, lattice field theory algorithms, continuum model building, accelerator beam quality, and detector design have led us to the threshold of developing a true understanding of the fundamental mechanisms of QCD and the ability to solve QCD, also at a long distance scale, quantitatively. However, such an understanding requires an extensive series of precise measurements, utilizing a hard electron-quark collision not only to access deep inelastic scattering processes, but also the more selective semiinclusive and deep exclusive processes.

This has led to the proposal of an Electron-Ion-Collider (EIC), a new machine with center-of-mass energies between 20 and $100 \mathrm{GeV}$, polarizations of both electron and proton (deuteron, ${ }^{3} \mathrm{He}$ beams) and luminosities up to $10^{35} \mathrm{~cm}^{-2} \mathrm{sec}^{-1}$. Two different approaches have been taken for the design of such a future Electron-Ion-Collider:

- One effort has focused on the utilization of the existing RHIC ion complex (eRHIC [2]). Adding either a $10 \mathrm{GeV}$ linear accelerator for polarized electrons or a small $10 \mathrm{GeV}$ polarized electron ring, luminosities of about $5 \cdot 10^{33}\left(5 \cdot 10^{32}\right) \mathrm{cm}^{-2} \mathrm{sec}^{-1}$ can be achieved. While the latter option uses existing accelerator technologies, a significant $R \& D$ effort is required to achieve the high intensity polarized electron current and energy recovery capability required in the design using a linear accelerator.

- In parallel, accelerator physicists at Jefferson Lab are pursuing a design which uses the CEBAF linear accelerator and requires the construction of a 30 to $225 \mathrm{GeV}$ ion storage ring [3]. This represents a more ambitious design concept to realize a luminosity of up to $8 \times 1034 \mathrm{~cm}-2 \mathrm{~s}-1$, using much higher collision frequencies and crab-crossing of colliding beams. In this concept, the booster rings, electron collider ring, and 
ion collider ring are designed as a figure 8, a design directly aimed at spin physics opportunities.

\section{The Structure of the Nucleon}

Great progress in our understanding of the structure of the nucleon has been made over the last decade at the HERA collider which has established the total dominance of gluons at low values of $x$. However, the same data also clearly the limit of the applicability of the conventional perturbative methods: at small values of $x$ and in the region of low $Q^{2}$, global parton distribution fitting algorithms result into gluon distributions smaller than the sea quark distributions and even turning negative. With the question still outstanding whether the gluons inside the proton can also behave as pre-existing, valence-like, constituents, or are rather the sole product of perturbative gluon Bremsstrahlung and gluon-gluon splitting processes, it is exactly this low $Q^{2}$ region where one would like to map the gluon content of the proton to study nucleon

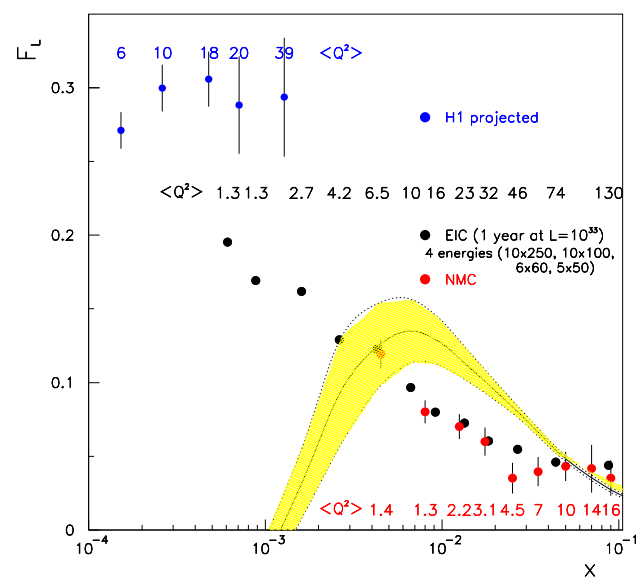

Figure 1: The longitudinal structure function $F_{L}$ expected from measurements at EIC in comparison with data from NMC and the expected results from HERA. structure.

Measurement of the longitudinal structure function $F_{L}$ would directly settle these issues. $F_{L}$ gives direct access to the gluon momentum distributions in the region of small $x$, where $F_{L} \approx \alpha_{s}\left(Q^{2}\right) x G\left(x, Q^{2}\right)$, and good access in the region of large $x$. The Electron-Ion Collider, with its variable energy scheme, would allow truely unprecedented measurements of $F_{L}$ (see Fig. 1). For the proton, this would render a substantial decrease of the uncertainties in $G\left(x, Q^{2}\right)$, especially in the region of interest, i.e. at low values of $x$ and moderate values of $Q^{2}$.

\section{The Spin Structure of the Nucleon}

One of the greatest successes of the Quark Parton Model is the description of the static properties of the nucleon and other baryons. Even though the naive quark parton model picture seems to perfect to account for the nucleon spin (3 valence quarks with spin $1 / 2$ arranged to result into a proton spin of $1 / 2$ ), deep inelastic scattering experiments have shown a very different picture. Over the last 20 years years the unpolarized (or spinaveraged) electron scattering measurements have been extended to precision spin-dependent measurements, rendering data on the $g_{1}$ structure function over a large range in $x$ and about one decade in $Q^{2}$. The major surprise from these results was that quarks and anti-quarks together carry only about $30 \%$ of the nucleon's spin. To then determine how the inner constituents of the nucleons, the valence quarks and the sea of quarks and gluons, conspire to provide the spin-1/2 of the nucleon, remains a challenging key issue in nuclear physics. 
Similar as in the unpolarized case, the dependence on $Q^{2}$ of the structure function $g_{1}$ has been used to constrain the gluon contribution to the proton spin. However, the precision and range in $Q^{2}$ of the existing data are far from optimal for this procedure to precisely determine the gluon spin distribution.

The proposed EIC will allow for precision measurements of the spin structure functions $g_{1}$, down to the smallest momentum fractions and over an unprecedented range of scales, as illustrated in Fig. /refFig:g1q2. This will provide crucial benchmark data to better pin down our present understanding of the precise contributions to the nucleon spin of quark and antiquark spin together. The increase range in $Q^{2}$ scales will similarly provide better constraints on the gluon contribution to the proton spin. The latter contributions can also be directly measured at the charm-quark mass scale with an EIC through low- $Q^{2}$ electroproduction of $D^{\circ}$ mesons. The high precision achievable in the determination of $\Delta G / G$ at EIC, using the latter method, is illustrated in Fig. 3.

With the realization that quarks and anti-quarks together only carry some 30\% of the proton spin, and gluons likely not completing this picture, orbital angular momentum of quarks and gluons has become a central issue in Nuclear Physics. To determine such orbital motion within the nucleons, a completely novel area of study, has been made possible with recent major theoretical breakthroughs that introduced more complete parton distribution functions termed "Generalized Parton Distributions" (GPDs) and "Transverse Momentum Dependent Parton Distributions" (TMDs), that both both contain information not only on the longitudinal momentum but also on the transverse spatial (or momentum) distribution of quarks and gluons in a fast moving hadron. As such, they are sensitive to the orbital motion of quarks and gluons, not accessible in inclusive scattering.

Determination of valence quark GPDs are the flagship of the physics program at the 12-GeV Upgrade at JLab. With EIC, it will be possible to extend the surveys of GPDs

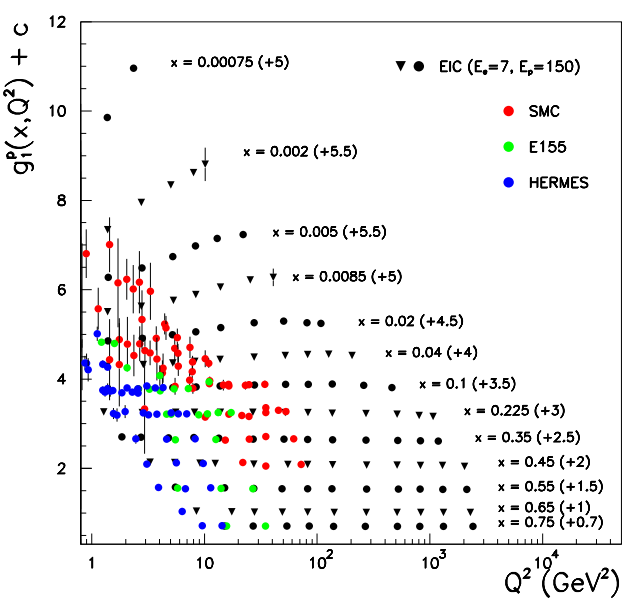

Figure 2: The expected precision on the polarized structure function $g_{1}\left(x, Q^{2}\right)$ at a future EIC in comparison with existing measurements.

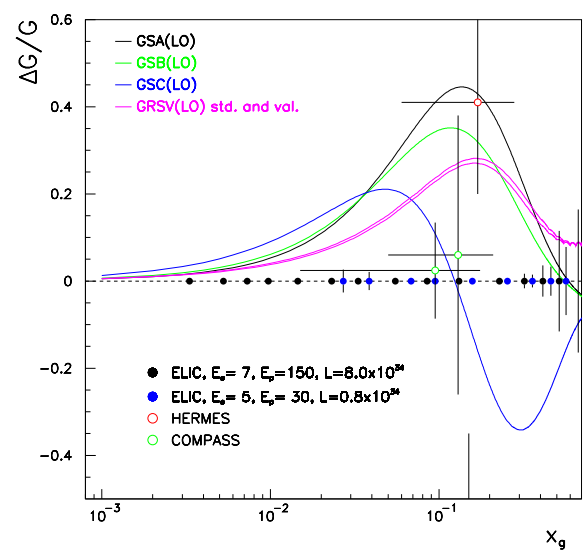

Figure 3: Measurement of the polarized gluon distribution from open charm production at the EIC in comparison with existing measurements and various models consistent with existing data. 
into the region where sea quarks and gluons abound. Electroproduction measurements of vector mesons, such as $\rho$ mesons and $\phi$ mesons, can be used to map the transverse spatial profile of gluons. Electroproduction measurements of charged pions can be extended to reach the limit, $Q^{2}>10 \mathrm{GeV}^{2}$, where one can safely believe access to GPDs is feasible for a quark-flavor separation.

Azimuthal distributions of final state hadrons in semi-inclusive deep inelastic scattering provide an independent window on the orbital motion of quarks, through the framework of TMDs. These describe transitions of nucleons with one polarization state to a quark with another polarization state. At the quark-gluon level, this probes the physics of initial and final state interactions. TMDs were introduced to explain the surprisingly large asymmetries found in hadronic reactions and, more recently, in semi-inclusive deep inelastic scattering experiments at HERMES, COMPASS, and JLab, with polarized targets.

In perturbative $\mathrm{QCD}$, which applies when the transverse momentum $p_{T}$ of the detected hadron is large compared to $\Lambda_{Q C D}$ (the scale where $\alpha_{s} \rightarrow \infty$ ), asymmetries vanish at leading twist level. The observed spin-dependent and spin-independent azimuthal asymmetries occur at $p_{T}$ below 1-2 GeV, not much larger than $\Lambda_{Q C D}$ or the typical quark-gluon transverse momenta of order $0.5 \mathrm{GeV}$. Thus, the measured asymmetries could arise from non-colinear parton (quark-gluon) or multi-parton correlations ("higher-twist" effects, suppressed at large $\left.p_{T}\right)$. Presently, the intrinsic transverse momentum of partons in the nucleon is at the root of most explanations of these non-zero azimuthal asymmetries. Measurements at EIC would be crucial, as they would extend measurements planned with the 12-GeV Upgrade at JLab into a region of large $p_{T}$, sufficiently large to provide an alternative "hard" scale for precise perturbative calculations.

\section{Summary}

The proposed Electron-Ion-Collider is the ideal machine to extend the measurements of polarized deep-inelastic scattering into a new level of kinematic coverage and precision. This will allow a precision determination of the gluon distribution at low $x$ and moderate $Q^{2}$, a measurement of the polarized sea quark distributions and a determination of the polarized gluon distribution over a very large range in $x$. Measurements of exclusive processes at sufficiently high values of $Q^{2}$ will enable to perform "gluon tomography" and thus a mapping of the transverse spatial profile of gluons. Together with measurements of transverse spin effects, these exclusive processes will determine the contribution of orbital momenta of quarks and gluons to the spin structure of the nucleon.

\section{Bibliography}

\section{References}

[1] Slides: http: //indico. cern. $\mathrm{ch} /$ contributionDisplay . py? contribId=258\&sessionId=10\&conf Id=9499

[2] eRHIC Zeroth Order Design Report, March 2004, edited by M. Farkhondeh and V. Ptitsyn.

[3] Zeroth-Order Design Report on Electron-Light Ion Collider at CEBAF, January 2007, edited by L. Merminga. 